\title{
Nefrite intersticial induzida por mesalazina em gêmeos monozigóticos portadores de doença inflamatória intestinal - relato de caso
}

\section{Mesalazine-induced interstitial nephritis in monozygotic twins with inflammatory bowel disease - case report}

José Célio Costa Lima Filho ${ }^{1}$. Maurício Yukio Ogawa ${ }^{2}$. Tacilla Hanny de Souza Andrade ${ }^{3}$. Sami de Andrade Cordeiro Gadelhaํ. Sonia Leite da Silva ${ }^{1}$. Claudia Maria Costa de Oliveira ${ }^{1}$. Anaiara Lucena Queiroz ${ }^{1}$.

1 Hospital Universitário Walter Cantídio (HUWC), Fortaleza, Ceará, Brasil. 2 Universidade Federal do Ceará (UFC), Fortaleza, Ceará, Brasil. 3 Universidade Estadual do Ceará (UECE), Fortaleza, Ceará, Brasil.

\section{RESUMO}

A doença inflamatória intestinal (DII) possui etiologia incerta, mas fatores ambientais, genéticos e imunológicos podem juntos justificar a patogênese, clínica e prognóstico desses pacientes. A manifestação clínica é diversa, sendo o acometimento renal um dos mais prevalentes, quando se exclui o trato gastrointestinal. Ademais, o papel genético na DII já foi mostrado em diversos trabalhos, principalmente em irmãos monozigóticos. A indução e manutenção do tratamento de casos leves/moderados de pacientes em remissão clínica apresenta boa eficácia com o uso de ácido 5-aminosalicílicos (mesalazina). Essa medicação, apesar de raro, também pode levar ao acometimento renal, com a nefrite intersticial sendo uma das principais etiologias. A seguir, descrevemos o caso de irmãos monozigóticos portadores de DII que desenvolveram nefrite intersticial em vigência de tratamento com mesalazina.

Palavras-chave: Doenças inflamatórias intestinais. Mesalazina. Ácidos aminossalicílicos. Nefrite intersticial. Gêmeos monozigóticos.

\section{ABSTRACT}

Inflammatory bowel disease (IBD) has uncertain etiology, but environmental, genetic and immunological factors may together justify the pathogenesis, clinical and prognosis of these patients. The clinical manifestation is diverse, with renal involvement being one of the most prevalent. In addition, the genetic role for IBD has already been shown in several studies, especially in monozygotic siblings. The induction and maintenance of the treatment of mild / moderate cases of patients in clinical remission shows good efficacy with the use of 5-aminosalicylic acid (mesalazine). This medication, although rare, may also have renal involvement with interstitial nephritis being one of the main etiologies. Next, we describe the case of monozygotic IBD siblings who developed interstitial nephritis during treatment with mesalazine.

Keywords: Inflammatory bowel diseases. Mesalazine. Aminosalicylic acids. Interstitial nephritis. Monozygotic twin.

Autor correspondente: José Célio Costa Lima Filho, Rua Coronel Nunes de Melo, 1315, Rodolfo Teófilo, Fortaleza, Ceará. CEP: 60430-160. Telefone: +55 85 99624-0916. E-mail: celiofilho_lima@yahoo.com.br

Conflito de interesses: Não há qualquer conflito de interesses por parte de qualquer um dos autores.

Recebido em: 21 Nov 2018; Revisado em: 04 Fev 2019; Aceito em: 04 Mai 2019. 


\section{INTRODUÇÃO}

Doença inflamatória intestinal (DII) corresponde a um processo inflamatório, cursando com quadros remitentes e recorrentes de diarreia sanguinolenta associado a dor abdominal, sendo um diagnóstico de exclusão, após descartadas causas metabólicas, tóxicas, isquêmicas, radiação. É dividida em dois grupos de patogenia, clínica e tratamento diferentes que são a Retocolite Ulcerativa Idiopática (RCUI) e a Doença de Chron (DC). A despeito dessa divisão, a etiologia de ambas parece ser ainda desconhecida, podendo ser de origem multifatorial: genética, imunológica e ambiental. ${ }^{1} \mathrm{O}$ risco é dez vezes maior para familiares de pacientes com DII. Ademais, irmãos monozigóticos parecem ter maior concordância do que irmãos dizigóticos no diagnóstico deRCUI. ${ }^{2,3}$

O envolvimento renal e urológico dos pacientes com DII ocorre em cerca de 4 a $23 \%$ dos pacientes, sendo o acometimento de parênquima renal considerado raro. ${ }^{4}$ Associado a isso, o tratamento da DII, principalmente nos casos de manutenção leve-moderado, é feito por meio do Ácido 5-aminossalicíco (Mesalazina), existindo diversos relatos de doença renal, apesar de prevalência rara, associada a esse fármaco, principalmente, nefrite intersticial e síndrome nefrótica. ${ }^{5}$

Descrevemos a seguir, um caso de dois irmãos monozigóticos portadores de Doença Inflamatória Intestinal sob uso de Mesalazina para controle da doença, com posterior desenvolvimento de nefrite intersticial.

\section{RELATO}

Caso 1 - Masculino, 23 anos, iniciou quadro de diarreia sanguinolenta associado a dor abdominal em 2015, sendo diagnosticado com RCUI e realizado tratamento de manutenção com Prednisona $60 \mathrm{mg} /$ dia por 06 meses e Mesalazina $2.400 \mathrm{mg} / \mathrm{dia}$. Apresentou elevação de creatinina cerca de um ano após início do tratamento, sendo suspenso a Mesalazina e encaminhado ao nosso serviço para acompanhamento. Exames (10/12/17) evidenciaram creatinina: $3,8 \mathrm{mg} / \mathrm{dl}$ (Taxa de filtração glomerular estimada pela fórmula CKD-EPI , $21 \mathrm{ml} / \mathrm{min} / 1,73 \mathrm{~m}^{2}$ ); Ureia: $80 \mathrm{mg}$ / dl;HbsAg: Não reagente (NR); Anti-Hbs: Reagente (Rea); Anti-HCV: NR; Anti-HAVIgM: NR; Anti-HAVIgG: Reag; Anti-HbCIgM: NR; Anti-HbCIgG: NR; Anti-HIV: NR; FAN: NR; Complemento (C3 e C4): Sem alterações; p-ANCA:NR; c-ANCA: NR; Índices hematimétricos sem alterações. Sumário de Urina (SU) (10/12/17): Ausência de hemoglobina e proteína. Ultrassom de vias urinárias (18/11/17): Normal. (Rim direito: 10,0 x 4,2 x 5,0 cm; Rim esquerdo: 10,1 x 4,5 x $5,1 \mathrm{~cm})$. Biópsia renal $(08 / 02 / 18)$ com infiltrado inflamatório linfoplasmocitário intersticial moderado associado a eosinófilos e moderada fibrose associada a atrofia tubular. Os túbulos exibem cilindros hialinos, focos de tubulite, e em revestimento, apagamento nuclear e descamação, com perda de bordas em escova. Tais alterações são sugestivas de nefrite tubulointersticial induzida por droga (Figura 1 e 2). Imunofluorescência indireta negativas para C3, C1q, fibrinogênio, $\operatorname{IgM}, \operatorname{IgG}, \operatorname{IgA}$, Kappa, Lambda.
Figura 1. Túbulos proximais com perda de borda em escova, descamação e cariólise, bem como túbulos contendo cilindros hialinos (HE, 400x).

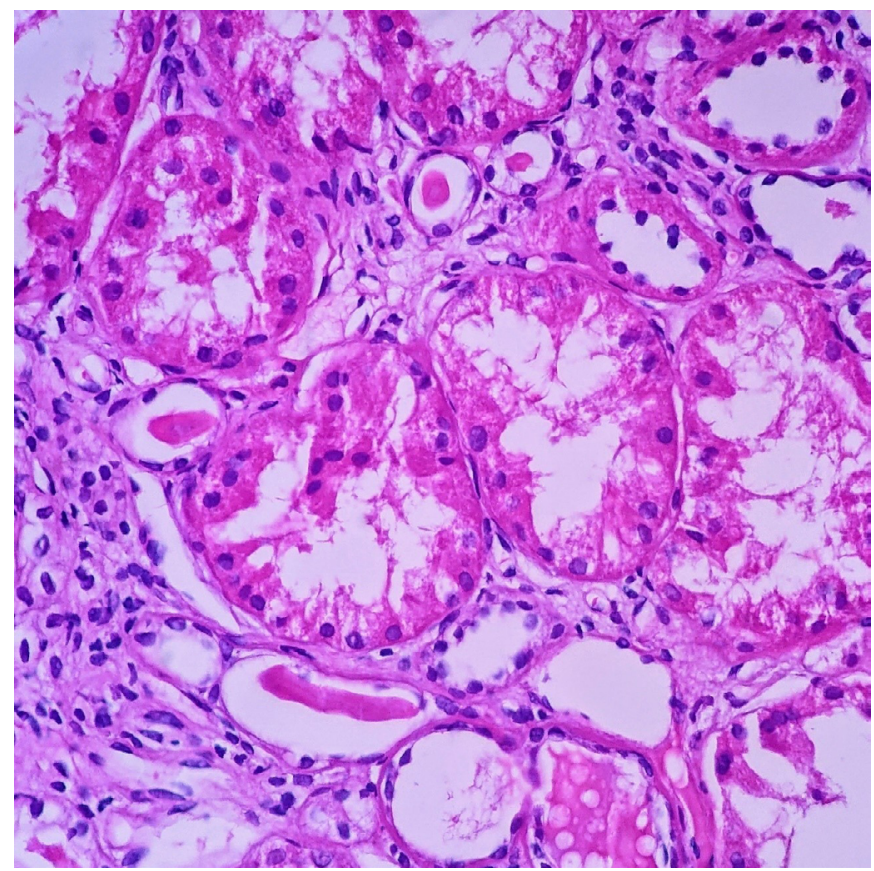

Figura 2. Infiltrado inflamatório intersticial linfocitário intenso com eosinófilos, além de túbulos atróficos (HE, 400x).

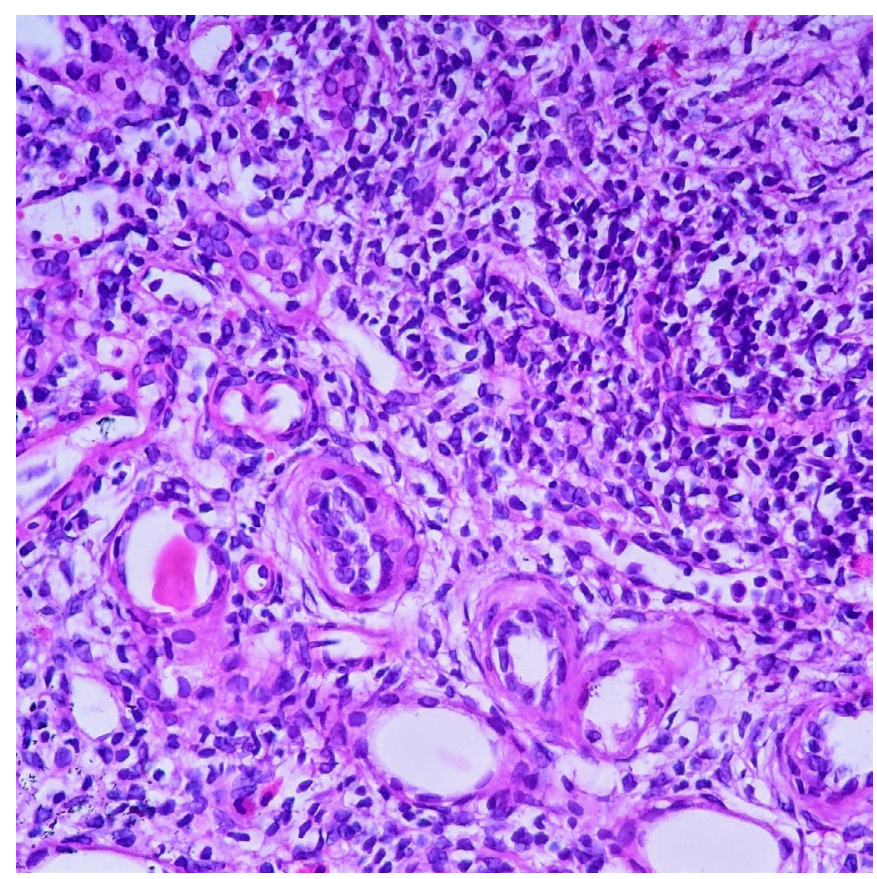

Caso 2 - Masculino, 23 anos, iniciou quadro de diarreia sanguinolenta associada a dor abdominal de forte intensidade em 2015, sendo diagnosticado com RCUI e realizado tratamento de manutenção com mesalazina $2.400 \mathrm{mg} /$ dia e prednisona $60 \mathrm{mg} / \mathrm{dia}$ por 06 meses. Apresentou elevação de creatinina cerca de seis meses após início do tratamento, 
sendo suspensa a medicação e encaminhado ao nosso serviço para acompanhamento. Exames (18/01/18): Ureia: $66 \mathrm{mg} /$ dl; Creatinina: 2,6 mg/dl (Taxa de filtração glomerular estimada pela fórmula CKD-EPI, $33 \mathrm{ml} / \mathrm{min} / 1,73 \mathrm{~m}^{2}$ ). Índices hematimétricos normais. HbsAg: NR; Anti-Hbs: Reagente (Rea); Anti-HCV: NR; Anti-HAVIgM: NR; Anti-HAVIgG: Reag; Anti-HbCIgM: NR; Anti-HbCIgG: NR; Anti-HIV: NR; FAN: NR; Complemento (C3 e C4): Sem alterações; p-ANCA:NR; c-ANCA: NR; SU (26/02/18): +/4+ de hemoglobina, bacteriúria discreta e ausência de proteínas. Biópsia renal (22/03/18) com infiltrado inflamatório intersticial linfocitário discreto com raros eosinófilos, além de túbulos proximais com descamação, cariólise, perda de borda em escova e alterações nucleares sugestivas de reparo em epitélio de revestimento compatível com nefrite intersticial leve por fármacos (Figura 3). Imunofluorescência indireta negativas para C3, C1q, Fibrinogênio, $\operatorname{IgM}$, IgA, IgG, Kappa, Lambda.

Figura 3. Infiltrado inflamatório intersticial linfocitário discreto com raros eosinófilos, além de túbulos proximais com descamação, cariólise, perda de borda em escova e alterações nucleares sugestivas de reparo em epitélio de revestimento (HE, 400x).

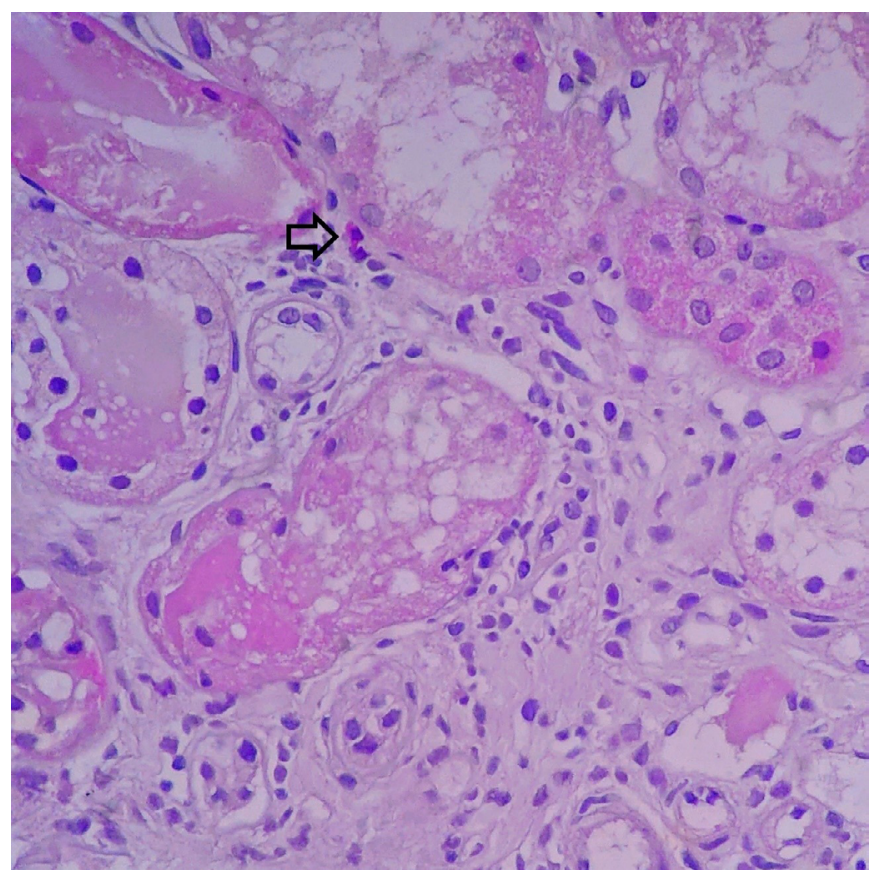

\section{DISCUSSÃO}

O envolvimento genético na gênese da DII ainda não é claro, mas diversos estudos epidemiológicos demonstram o seu papel. ${ }^{1,2,3}$ A alta concordância entre gêmeos monozigóticos em relação aos dizigóticos reforça tal caráter. ${ }^{3}$ Alguns estudos sugerem ainda que esse componente genético é mais associado à etiologia da RCUI comparado à DC. ${ }^{6} \mathrm{~A}$ correlação temporal da sintomatologia evidenciada mostrada no relato costuma ser o mais prevalente, apesar de existir relato de diferença de décadas entre a sintomatologia de gêmeos. ${ }^{6}$
As complicações renais ou urológicos na DII não são raras, com prevalência de 4 a $23 \%$, sendo as mais comuns: litíase renal, fístula enterovesical ou obstrução ureteral. ${ }^{4}$ Entre as complicações renais, as mais prevalentes são lesão renal aguda, anormalidades glomerulares e proteinúria tubular. ${ }^{5} \mathrm{Um}$ estudo retrospectivo de biópsias renais de pacientes com DII mostrou a Nefropatia associada a IgA e Nefrite intersticial como as mais prevalentes nesses pacientes. ${ }^{7} \mathrm{O}$ risco de tais complicações é potencializado pelo uso de medicamentos, em especial, o ácido 5-aminosalicílico. ${ }^{5}$

O ácido 5-aminosalicílico (mesalazina) foi desenvolvida a partir da sulfasalazina em 1977, sendo uma medicação considerada eficaz para a indução e manutenção da remissão clínica em pacientes com DII, proporcionando um melhor perfil de tolerabilidade em relação a seu antecessor. ${ }^{8,9,10} \mathrm{~A}$ despeito disso, os efeitos colaterais renais da mesalazina, apesar de raros, podem impactar na morbidade dos pacientes, inclusive com risco de evolução para doença renal terminal. ${ }^{8}$ Tais efeitos parecem não ter relação com a posologia e o tempo administrado, acreditando-se ter característica idiossincrática. ${ }^{8,11}$

O acometimento renal mais prevalente da mesalazina é a nefrite intersticial, seja agudo ou crônico, mas também é associado ao seu uso: glomerulonefrite, nefropatia por lesão mínima com síndrome nefrótica e há até mesmo relato de diabetes insipidus nefrogênica. ${ }^{12,13}$ Alguns autores questionam se o substrato genético também não teria influência para o desenvolvimento dessas lesões. Um trabalho sugeriu que alterações de alelos do sistema leucocitário humano (HLA) poderia ser um fator de risco, mas com baixa frequência e utilidade clínica limitada. ${ }^{14}$

O diagnóstico desses pacientes consiste na suspeição clínica em pacientes sob uso de mesalazina e que apresentam queda da função renal. A despeito disso, ainda é motivo de controvérsia a relação custo-benefício de avaliação rotineira da função renal. ${ }^{8,14} \mathrm{O}$ tratamento desses pacientes consiste na suspensão da medicação assim que identificado alterações, com a maioria dos pacientes apresentando recuperação da função renal. O prognóstico desses pacientes parece ter grande relação com o tempo de diagnóstico, apresentando desfecho mais favorável aqueles em que o diagnóstico é feito dentro de 10 meses. ${ }^{8}$ Cerca de $10 \%$ desses pacientes acabam por evoluir para doença renal terminal. ${ }^{11} \mathrm{O}$ paciente do caso 1 evoluiu para doença em estágio terminal, enquanto o caso 2 apresentou melhora parcial da função renal após suspensão da medicação, com taxa de filtração glomerular, segundo a fórmula CKD-EPI, aumentando para $41 \mathrm{ml} /$ $\min / 1,73 \mathrm{~m}^{2}$.

\section{CONCLUSÃO}

A DII, a despeito das incertezas sobre sua etiologia, apresenta uma predisposição genética, vista em vários estudos epidemiológicos. A prevalência entre irmãos monozigóticos reforça essa possibilidade. ${ }^{6}$ A DII associado ao uso de mesalazina pode culminar em uma diversidade de patologias 
renais, sendo a nefrite intersticial uma das mais prevalentes. ${ }^{7}$ Os casos apresentados são uma valorosa contribuição à literatura médica, tendo em vista que só foi encontrado um relato semelhante. ${ }^{15}$

\section{REFERÊNCIAS}

1, Xia B, Crusius JB, Meuwissen SG, Peña AS. Inflammatory bowel disease: definition, epidemiology, etiologic aspects, and immunogenetic studies. World J Gastroenterol. 1998;4(1-6):446-58.

2. Clark SJ, Saag MS, Decker DW, Campbell-Hill S, Roberson JL, Veldkamp PJ, et al. Familial ocurrence of inflammatory bowel disease. N Engl J Med. 1991;324(14):954-60.

3. Horiya M, Kakizaki S, Teshigawara K, Kikuchi Y, Hashida T, Tomizawa Y, et al. Concordance of ulcerative colitis in monozygotic twin sisters. World J Gastroenterol. 2005;11(47):7547-9.

4. Pardi DS, Tremaine WJ, Sandborn WJ, McCarthy JT. Renal and urologic complications of inflammatory bowel disease. Am J Gastroenterol. 1998;93(4):504-14.

5. Van Staa TP, Travis S, Leufkens HG, Logan RF. 5-Aminosalicylic acids and the risk of renal disease: a large British epidemiologic study. Gastroenterology. 2004;126(7):1733-9.

6. Cleymans J, Thews RL. Inflammatory bowel disease in a Swedish twin cohort: a long-term follow-up of concordance and clinical characteristics. Zeitschrift für Phys C Part Fields. 1990;45(3):391-7.

7. Ambruzs JM, Walker PD, Larsen CP. The histopathologic spectrum of kidney biopsies in patients with inflammatory bowel disease. Clin J Am Soc Nephrol. 2014;9(2):265-70.

8. Gisbert JP, González-Lama Y, Maté J. 5-Aminosalicylates and

\section{CONSIDERAÇÕES ÉTICAS}

Todo o trabalho foi conduzido de forma ética seguindo critérios estabelecidos pela Declaração de Helsinki com as suas modificações (Bull World Health Organ. 2001;79:373-374).

renal function in inflammatory bowel disease: a systematic review. Inflamm Bowel Dis. 2007;13(5):629-38.

9. Gisbert JP, Gomollón F, Maté J, Pajares JM. Role of 5-aminosalicylic acid (5-ASA) in treatment of inflammatory bowel disease: a systematic review. Dig Dis Sci. 2002;47(3):471-88.

10. Chester AC, Diamond LH, Schreiner GE. Hypersensitivity to salicylazosulfapyridine renal and hepatic toxic reaction. 1978;138:1138-9.

11. Arend LJ, Springate JE. Interstitial nephritis from mesalazine: case report and literature review. Pediatr Nephrol. 2004;19(5):550-3.

12. Oikonomou KA, Kapsoritakis AN, Stefanidis I, Potamianos SP. Drug-induced nephrotoxicity in inflammatory bowel disease. Nephron Clin Pract. 2011;119(2):c89-c96.

13. Skalova S, Dedek P, Pozler O, Podhola M. Mesalazine-induced interstitial nephritis. Ren Fail. 2009;31(2):159-61.

14. Heap GA, Weedon M, Edney N, Bewshea C, Annese V, Beckly $\mathrm{J}$, et al. Clinical features and HLA association of 5-Aminosalicylate (5-ASA) induced nephrotoxicity in inflammatory bowel disease. J Crohns Colitis. 2016;10(2):149-58

15. Sato H, Umemura K, Yamamoto T, Sato H. Interstitial nephritis associated with ulcerative colitis in monozygotic twins. BMJ Case Rep. 2017;2017:10-3.

\section{Como citar:}

Lima JC Filho, Ogawa MY, Andrade TH, Gadelha SA, Silva SL, Oliveira CM, et al. Nefrite intersticial induzida por mesalazina em gêmeos monozigóticos portadores de doença inflamatória intestinal - relato de caso. Rev Med UFC. 2019 out-dez;59(4):83-86. 